\title{
La représentation du sucre à Bruxelles : sociohistoire des pratiques de lobbying auprès des instances européennes depuis le début du $\mathrm{XX}^{\mathrm{e}}$ siècle
}

\author{
Marie HRABANSKI \\ UMR ART Dev, CIRAD, 73 rue Jean-François Breton, 34398 Montpellier cedex 5, France \\ e-mail: marie.hrabanski@cirad.fr
}

Résumé - L'article porte sur des formes de lobbying pratiquées auprès des instances européennes par les organisations professionnelles agricoles de la filière du sucre et leurs représentants, du début du XXe siècle jusqu’à aujourd'hui. L'étude dessine trois périodes distinctes. La première, avant la structuration politique de l'Europe, a jeté les bases de la période suivante, qui commence en 1967 au moment de la création de la Communauté économique européenne (CEE), et qui se caractérise par une cogestion forte entre les instances communautaires et les représentants de la filière. La troisième période s'intéresse aux transformations du lobbying à partir des années 1980, au moment de l'entrée dans l'ère de la globalisation et du pluralisme. Malgré les évolutions du système d'intermédiation des intérêts de l'Union européenne (1992), les représentants de la filière vont poursuivre leur stratégie d'influence, basée notamment sur les relations privilégiées qu'ils entretiennent avec des représentants de la Commission européenne. Cette dernière période souligne l'importance du carnet d'adresses dans les processus politiques et se caractérise également par un recours accru à l'expertise par la Commission.

Mots-clés : groupes d'intérêt, lobbying, agriculture, Europe, politiques publiques, expertise

\section{Representation of sugar in Brussels: Historical sociology of lobbying practices in the European institutions since the early $\mathrm{XX}^{\text {th }}$ century}

Summary - This article deals with the practices of lobbying made by some agricultural interest groups and their representatives in the "sugar sector", at the European level, since the early $20^{\text {th }}$ century until today. The study draws three distinct periods. The first shows bow an international organization of European Beet Growers has been structured and represented its interests at the international level even before the political community of Europe. This first period laid the foundation for the next one, which begins at the creation of the EEC in 1967, and is characterized by a strong co-management between the authorities and industry representatives. The third period is concerned with transformations of lobbying from the 1980s, when entering the era of globalization and pluralism. Despite changes in the system of interest intermediation of the European Union (1992), the representatives of the sector will continue their strategies, based on the special relationship they have with representatives of the European Commission. At the same time, this last period highlights the importance of the address book in political processes, and is also characterized by the use of expertise by the Commission.

Keywords: interest groups, lobbying, agriculture, Europe, policy making, expertise

Classification JEL : F02, J51 


\section{Introduction}

Depuis les années 1990, les controverses sur le lobbying se multiplient en France et en Europe : la Commission européenne, en proposant un livre blanc sur la gouvernance européenne (Commission européenne, 2001) met en évidence la volonté institutionnelle d'éclaircir les liens entre les groupes d'intérêt et les instances décisionnelles européennes, afin de répondre aux critiques régulièrement émises sur l'opacité du système politique communautaire. De même, au printemps 2008, dans le cadre de sa politique de transparence, l'Union européenne (UE) a invité les «lobbies » et think tank à se déclarer sur un registre facultatif, destiné à comptabiliser et à rendre transparents les financements des groupes d'intérêt œuvrant à Bruxelles. Elle essaie de cette façon de réguler les pratiques professionnelles des représentants d'intérêts. De même, les associations européennes - European Public Affairs Consultancies Association (EPACA), Society of European Affairs Professionals (SEAP ${ }^{1}$ ) - et nationales, telles que l'AFCL ${ }^{2}$ (association française des conseils en lobbying), tentent de mettre en place des chartes, non contraignantes, de «bonnes pratiques» professionnelles. Ces initiatives récentes correspondent surtout à une institutionnalisation (Freidson, 1998) de la profession de lobbyiste, encouragée par l'initiative de la Commission. Elles questionnent toutefois plus largement la nature des pratiques professionnelles des lobbyistes.

Ce type de questionnement est peu fréquent dans la littérature. Hélène Michel, en analysant les travaux ayant trait au lobbying, montre que toute une série d'ouvrages tend à construire une image partielle et partiale des lobbies et du lobbying (Michel, 2003 et 2005). Vecteurs d'une démocratisation du processus décisionnel européen pour les uns, à l'assaut de l'Europe pour d'autres, cette littérature suit et alimente surtout les débats lancés par les institutions européennes, à savoir les questions de transparence et de régulation du lobbying. Ce type d'approche tend à naturaliser le lobbying, or comme l'a montré Hélène Michel, cette pratique recouvre des réalités hétérogènes. D’autres travaux s'y sont intéressés, en termes de système d'intermédiation des intérêts (pluraliste, néocorporatiste...) (Ayberk et Schenker, 1998 ; Richardson, 1993) mais tendent à passer sous silence le rôle des acteurs dans les processus politiques. On se propose ici de compléter ces analyses en articulant les approches micro et macrosociologiques. Autrement dit, pour comprendre la représentation du sucre auprès des instances de l'Union européenne, il convient de s'intéresser aussi aux représentants de la filière à Bruxelles. Il s'agira notamment de montrer que les pratiques de lobbying sont construites socialement et historiquement, en fonction du contexte organisationnel et politique dans lequel le lobbying est mené. Notre article souhaite, dans cette perspective, examiner l'évolution des pratiques de lobbying, dans le secteur du sucre, depuis le début du $\mathrm{XX}^{\mathrm{e}}$ siècle, à savoir avant même l'existence de l'UE. Le choix de cette filière repose sur deux critères: le secteur est à la fois historiquement implanté à l'échelle européenne et représente surtout un poids économique considérable. Les 160000 betteraviers européens issus des 21 pays producteurs de l'UE sont représentés dans l'espace politique communautaire via la Confédération internationale des betteraviers européen (CIBE), et la Confédération européenne des fabricants de sucre (CEFS) représente quelques 56 entreprises et près d'une

${ }^{1}$ L'EPACA et la SEAP ont été respectivement créées en 2005 et 1997.

2 L'AFCL a été créée en 1991. 
centaine de sucreries ${ }^{3}$. Elles sont de puissants groupes d'intérêt et cumulent des ressources organisationnelles, financières et politiques (Grossman et Saurugger, 2006): l'UE est le 3 e producteur mondial de sucre, derrière le Brésil et l'Inde. Elle est également le premier consommateur et le deuxième exportateur mondial, ainsi que l'un des trois principaux importateurs.

L'analyse diachronique privilégiée ici nous pousse à contextualiser l'action des représentants de la filière. Dans quelle mesure la dimension européenne des stratégies de pression des représentants de la filière "sucre" est-elle nouvelle? Si, comme notre première hypothèse le suggère, l'implication des associations spécialisées sur les questions internationales est très ancienne, voire structurante de leur identité et de leurs pratiques, dans quelle mesure alors l'introduction et la consolidation politique de l'espace communautaire ont-elles bouleversé les modalités d'action des associations agricoles, et les pratiques professionnelles des représentants? Et aujourd'hui, quelles sont les différentes formes du lobbying menées par les acteurs de la filière? H. Delorme estime ainsi que depuis les années 1990, au niveau européen, le corporatisme agricole a cédé la place au lobbying agro-alimentaire (Delorme, 2002). A quelles pratiques correspond ce lobbying et que cela révèle-t-il du système politique européen? Nous avançons une seconde hypothèse selon laquelle les professionnels du sucre adaptent leur stratégie aux demandes de la Commission et conforment leurs pratiques aux attentes institutionnelles de l'UE, infirmant ainsi la thèse selon laquelle le lobbying constituerait une certaine forme de privatisation de l'action publique (Demortain, 2005). Les transformations des pratiques de lobbying pourraient, dans cette perspective, éclairer l'évolution de l'UE.

Pour étayer nos hypothèses, l'article repose sur une analyse des archives de la CIBE, une dizaine d'entretiens semi-directifs auprès des professionnels du sucre ainsi qu'une enquête ethnographique au sein des groupes consultatifs «sucre » de l'UE. A la croisée de la sociologie des pratiques professionnelles et de la sociologie politique, l'article propose une lecture en plusieurs étapes. Une analyse historique des conditions de création des organisations transnationales de la filière sucre et des grandes phases de leur développement permettra de souligner la précocité de l'implication des groupes d'intérêt sur les questions internationales. Ensuite, à travers l'analyse des différentes pratiques de lobbying, il conviendra de s'intéresser à leur évolution. Opaques pendant les années 1980 et l'entrée dans l'ère de la globalisation, elles vont peu à peu se juxtaposer aux modes de représentation des intérêts et aux codes rhétoriques promus par l'UE, notamment via l'expertise.

\section{Transformations des relations entre les organisations professionnelles du sucre et les instances européennes depuis le début du $\mathrm{XX}^{\mathrm{e}}$ siècle}

L'analyse historique proposée permet de recontextualiser les modes de représentation des groupes d'intérêt «sucre ». Des conditions d'émergence spécifiques

\footnotetext{
3 Chiffres CEFS, 2011
} 
ont favorisé leur implication précoce sur les questions européennes, et ce dans le cadre de relations de type corporatiste. Forts de ces savoir-faire, le groupe d'intérêt transnational va investir aisément les instances décisionnelles internationales et notamment la Société des Nations (SDN), puis la CEE et l'UE. Ainsi l'objectif de cette première partie historique est de montrer que les relations entre les groupes d'intérêt et les instances européennes sont anciennes, et que ce ne sont pas les institutions qui suscitent de telles relations mais qu'elles leur préexistent.

\subsection{Cogestion et constitution d'une élite européenne de la filière sucre}

Peu d'études historiques se sont intéressées au type de relations qu'entretenaient les milieux économiques avec les instances décisionnelles avant la construction européenne. Dans les années 1920, deux types de structures témoignent de la montée en puissance des préoccupations européennes. D'un côté, on observe des mouvements chargés de représenter le monde des grandes entreprises (Buissière, 2006). Ces structures défendaient leurs positions essentiellement auprès des pouvoirs publics à travers leurs sections nationales. Leurs actions à l'échelle de la SDN se limitaient à "des activités de concertation, d'élaboration de proposition, de diffusion». Eric Buissière met en évidence les filiations entre ces premiers mouvements et les organisations patronales des décennies suivantes. A côté de ces mouvements, vont émerger des structures, parfois qualifiées de cartel (Barjot, 1994), comme la Confédération internationale des betteraviers (CIBE) ou l'Entente internationale de l'acier, chargées d'organiser le marché européen et les rapports d'une profession ou d'une filière donnée avec la concurrence internationale.

Ainsi, lorsqu'en 1925, la Confédération internationale des betteraviers européens s'est constituée, il s'agissait d'instaurer "une entente durable et organisée entre les différents producteurs de betteraves» (Cayre, 1982). Derrière cet objectif lissé, plusieurs facteurs autrement plus essentiels semblent avoir favorisé l'émergence de l'organisation transnationale. Comme l'expliquent D. Della Porta et S. Tarrow (2005), «les conditions macro structurelles ou culturelles ne suffisent pas à produire une mobilisation, mais c'est lorsque des ressources et des opportunités propres aux groupes émergent ensemble, que les intérêts et les incitations peuvent donner lieu à une action collective concertée ». De même, l'existence de problèmes agricoles européens ne suffit pas à déclencher une action collective. C'est donc en nous éloignant de cette dimension déterministe que nous avons souhaité interroger les conditions favorables à la constitution de la CIBE. Cette dernière, malgré son appellation, rassemblait les fabricants de sucre et les producteurs européens dans une même structure. Il s'agissait avant tout, pour eux, de lutter contre la concurrence, jugée trop forte, des sucres de canne. En effet, la production de sucre de canne, peu touchée par la Première Guerre mondiale et encouragée par une forte hausse des prix, se développait considérablement, doublant entre 1913 et 1929, tandis que les terres en Europe avaient servi de champ de bataille au conflit. Partageant des intérêts assez proches, les différents représentants nationaux de la filière espéraient peser sur les négociations internationales et, via la CIBE, devenir des interlocuteurs crédibles aux yeux de la SDN.

Par ailleurs, dans la lignée des conclusions de P. Barral (Barral, 1968), la filière sucre, après la Première Guerre, semble se transformer et abandonner de plus en plus les luttes dispersées pour l'action collective (période de 1860 à 1914), tout en adoptant 
un système de représentation politique dominé par l'élite agricole de la profession. Issue de la grande bourgeoisie rurale du début du $\mathrm{XX}^{\mathrm{e}}$ siècle, l'élite européenne de la filière sucrière partageait des propriétés sociales tout à fait favorables à leur rapprochement. A ce titre, l'analyse des archives internes de la CIBE souligne les proximités sociales des différents représentants nationaux. Grâce à plusieurs documents internes des organisations professionnelles du sucre, on obtient des éléments sur la biographie des responsables de la CIBE et du Comité européen des fabricants de sucre (CEFS) depuis leur création, comme l'illustrent ces trois exemples:

Eléments de biographie d'un dirigeant polonais de la CIBE, tirés de Henry Cayre (1982). "C'était un homme d'une grande qualité, très cultivé qui parlait aussi bien l'anglais que l'italien ou le français, en plus de sa langue maternelle. Le Baron de X était issu d'une grande famille de la noblesse polonaise et son rôle en Europe fut éminent. Animateur dès la première beure de la Confédération internationale des betteraviers, il fut de toutes les négociations betteravières, sucrières et alcoolières. Quel déchirement cela a été pour eux et pour nous lorsque, après 1945, le régime communiste nous priva de nos échanges!».

Eléments de biographie d'un dirigeant italien de la CIBE, puis du CEFS (Cayre, 1982). "Eclectique, aucun domaine d'activité ne lui était indifférent et ce dans un élan toujours renouvelé. Il avait fait de brillantes études, que ce soit la génétique, l'agronomie, l'économie politique, tout lui était cher et constituait son terrain d'action. Gino de X, quel bomme cultivé, merveilleux, plein de cceur, ce fut pour moi un grand frère, qui me fit découvrir l'Italie que j'ai la grande chance de connaître aussi bien que mon pays. Nous fîmes reviure la CIBE tous les deux, après nous être rencontrés dans un café où nous avions rendez-vous à Venise en août 1946 ».

Eléments de biographie d'un responsable français de la CIBE, tirés de Eloge de Monsieur X (France) par Arthur Boursier (Boursier, 1983). "Frais émoulu de l'Université, avec une double formation d'économiste et de juriste, on aurait pu penser que vous étiez imbu des théories à la mode de Lord John Maynard Keynes !... Ce fut une surprise que de vous voir plus proches des physiocrates, chers à M. Turgot, pour qui la source essentielle de la richesse ne pouvait provenir que de l'agriculture. (...) Vous maitrisez plusieurs langues européennes et nous ne répéterons jamais assez combien votre action à la tête de la CIBE, dans les années 1950, a été déterminante (...) Vous avez compris dans les premiers, combien il était important d'informer de nos problèmes les milieux législatifs et parlementaires. Vos liaisons avec les instances politiques et les liens d'amitié que vous avez su nouer avec de nombreux membres du Parlement contribuèrent à doter l'économie betteravière d'une protection minimale».

L'analyse de ces biographies souligne à quel point les responsables de la filière sucre appartenaient à l'élite agricole de l'époque. Grands propriétaires fonciers pour les uns, fermiers liés à l'industrie sucrière pour d'autres, ces représentants rassemblaient les dispositions à l'international ainsi que les capitaux économiques, sociaux et culturels, 
désormais bien identifiés par la sociologie des élites (Wagner, 1998; Pinçon and Pinçon-Charlot, 1997) nécessaires à leur rapprochement. Cet engagement européen était couplé à un ancrage national fort: les représentants œuvraient en même temps auprès de leur Etat pour l'obtention de mesures de protection de la production betteravière domestique, afin de se «protéger» du sucre de canne, et pour l'organisation des relations entre sucriers et producteurs. Il parait toutefois bien difficile de chiffrer le nombre de dirigeants des organisations professionnelles de la filière sucre de cette période. Les archives mentionnent uniquement le rôle des présidents et secrétaires généraux, et ne font pas état de salariés.

Le contexte politique de cette époque était également favorable à l'émergence de la CIBE. En effet, peu à peu, en France comme dans les autres grands pays producteurs de betterave (Allemagne), la filière sucre se structure au niveau national et s'inscrit rapidement dans une relation de type corporatiste avec l'Etat (Muller, 1980). P. Barral (1968) souligne à cet effet que la société paysanne s'est inscrite jusqu'à la Première Guerre mondiale dans une logique de distinction et de séparation du reste de la société ; toutefois, après 1918 , elle va chercher au contraire une défense à l'intérieur du consensus collectif, et promouvoir ainsi la cogestion du secteur agricole. Les représentants français de la filière sucre ont été particulièrement actifs dans la création de la CIBE et ont œuvré à la cogestion du secteur betteravier au niveau international, suite à la création de la Société des Nations en 1919. De cette façon, la prise en compte des questions internationales et leur règlement dans des relations de proximité, de type corporatiste, est inhérente à la constitution du groupe d'intérêt transnational.

\subsection{La permanence des stratégies d'influence du groupe d'intérêt transnational dans la CEE}

Pendant l'entre-deux-guerres, en France et plus généralement en Europe (Hervieu et Lagrave, 1992), les intérêts des planteurs et des fabricants étaient largement enchevêtrés, et les représentants professionnels les plus compétitifs ont noué des rapports privilégiés et fermés avec les pouvoirs publics. Chacun a ainsi puisé, dans ces relations, l'information et les moyens de légitimer ses actions (Hervieu, 1996). Après la Seconde Guerre mondiale, le marché commun devient une des préoccupations majeures des représentants de la filière sucre au niveau national et au niveau européen. Favorables à un prix unique du sucre en Europe, les intérêts des fabricants commencent à se distinguer nettement de ceux des producteurs, ce qui n'était encore que peu le cas auparavant. La création du Comité européen des fabricants de sucre (CEFS), en 1954, va permettre le rassemblement de l'élite industrielle de la filière sucre d'Europe de l'Ouest, la situation géopolitique à l'est de l'Europe ayant écarté ses représentants.

Malgré la création du CEFS, le compromis entre les producteurs et les fabricants va émerger rapidement, puisqu'en 1959, ils élaborent conjointement une position commune favorable au régime des quotas de production (Boursier, 1983). Ce système prévalait entre 1930 et 1939 lorsqu'il fallait gérer l'abondance. De 1949 à 1959, le problème était inversé, puisqu'il fallait faire face à la pénurie. Ce sont les filières, inspirées par les modes de régulation qui s'appliquaient déjà pour d'autres matières premières, qui vont réussir à maintenir un système de quotas chargé d'encourager la 
production. Ainsi bien avant la CEE (1957), la filière sucre était parvenue à imposer une réponse spécifique au "problème public » de la pénurie alimentaire, un cadre d'interprétation du monde (Muller, 2000): il s'agissait d'encourager la productivité agricole (Servolin, 1989). Tandis que les syndicats nationaux à vocation générale se sont approprié les questions dites politiques, liées notamment à la modernisation des structures agricoles, les questions dites «économiques » ont été laissées à la charge des filières agricoles (Fouilleux, 2003; Pesche, 2000). Les filières se sont facilement converties à un mode de gestion communautaire qui ne dénotait pas avec le fonctionnement précédent. La construction européenne ne va pas affaiblir l'audience de la filière sucre puisque celle-ci va réinvestir le savoir-faire et les relations acquises avant la CEE, au sein de la DG-VI (Direction générale de l'Agriculture, DG Agri).

Ainsi, à partir de 1962, les orientations agricoles dépendent principalement de la politique agricole commune menée par la CEE (1957). La majorité des décisions sont prises à Bruxelles sur proposition de la Commission européenne, et leur mise en œuvre est négociée en partie au niveau national. Cependant cette période n'introduit pas de rupture dans les stratégies d'influence des betteraviers et des fabricants. Le corporatisme de l'appareil européen de représentation des agriculteurs et des industriels, bâti sur un « référentiel » partagé entre les décideurs et les représentants professionnels (Delorme, 2002), va permettre à la filière de poursuivre ses stratégies de représentation. Fervents militants de l'Europe, les organisations professionnelles de la filière sucre vont aussi défendre des intérêts économiques liés à la mise en place d'une Europe supranationale. Ainsi, lors de la conception de l'OCM sucre en 1968, sous la pression des betteraviers et plus encore des industriels, le principe des quotas fut une nouvelle fois reconduit et, cette fois, adopté par la CEE. L'étude des archives de la CIBE rassemblées par Henry Cayre (1982) souligne l'étroitesse des relations entre les responsables du CEFS et les différents commissaires européens. Lors des interviews effectuées à la DG Agri, les différents fonctionnaires ont confirmé l'empreinte de la CEFS sur le fonctionnement de l'OCM, allant même jusqu'à évoquer la prégnance des fabricants européens sur la division sucre.

Entretien DG Agri avril 2006 : "Dans les années 60-70-80, ils étaient comme chez eux ici, ils entraient ils sortaient, ils faisaient un peu ce qu'ils voulaient, ce qui les arrangeait, et ça... ça a duré un bon moment jusqu'au début des années quatre-vingt dix, et puis après ça a changé quand même, on a dit stop... Mais c'est vrai que si vous en discutez un peu avec les anciens, on vous dira que c'était incroyable, c'est quasiment eux qui rédigeaient les notes des fonctionnaires... Aujourd'bui, oui, ils sont encore là, tenez, il y en a un de chez eux qui vient de passer dans le couloir, mais ce n'est plus pareil... »

Lorsque la CEE est créée en 1967, les betteraviers et les fabricants de sucre, forts de leurs compétences techniques, de leur connaissance du marché international, des relais locaux nécessaires à la mise en place concrète des mesures d'encadrement de la filière, et bénéficiant d'une certaine légitimité historique, vont poursuivre la cogestion du secteur avec les fonctionnaires européens. C'est à partir de cette période que la CIBE et le CEFS recrutent leurs premiers salariés et, notamment, des analystes spécialisés en économie, chargés de produire et de diffuser de l'information sur la filière. Leur nombre reste toutefois assez limité jusqu'au début des années 1980. L'historicité des liens entre les 
organisations de la filière sucre et les institutions supranationales avant la construction vont leur permettre "naturellement » de poursuivre leurs activités de représentation au sein de la CEE et d'être ainsi étroitement liées à la gestion de la politique agricole commune (PAC). Aussi, jusqu’à la fin des années 1970, la CIBE, et à travers elle les associations nationales les plus puissantes (la Confédération générale des planteurs de betteraves (CGB) pour la France, l'Arbeitsgemeinschaft Deutscher Rinderzüichter (ADR) pour l'Allemagne), a plutôt été invité à transmettre ses positions à la DG Agri qu'à lui soumettre. A partir des années 1980, plusieurs types de contraintes vont peser sur les rapports entre les décideurs européens et les représentants de la filière sucre, sans toutefois les transformer profondément. L'analyse des pratiques de représentation des intérêts et, notamment, des réseaux d'influence dont les représentants de la filière bénéficient, soulignent la proximité encore effective entre la Commission et les groupes d'intérêt dans le secteur du sucre.

\section{La représentation des intérêts de la filière sucre dans l'ère de la globalisation : la mobilisation du carnet d'adresses comme ressource politique}

Nous l'avons vu, l'implication des associations spécialisées dans les questions internationales est très ancienne. Elle semble même avoir structuré leur identité et leurs pratiques. Toutefois, à partir de 1962, les orientations agricoles dépendent principalement de la PAC menée par la CEE (1967), puis par l'Union européenne (1992). La majorité des décisions sont prises à Bruxelles sur proposition de la Commission européenne, et leur mise en œuvre est négociée en partie au niveau national. Engagées de longue date dans la défense de leurs intérêts à l'échelle internationale, les associations spécialisées vont renforcer leur intervention au niveau communautaire, tout en maintenant leur stratégie de mobilisation à l'échelle nationale (Pesche et Hrabanski, 2010). Ce processus d'européanisation de la représentation agricole s'inscrit depuis les années 1980 dans le cadre plus général de la globalisation. Les relations de cogestion, qui ont largement dominé le système d'intermédiation des intérêts agricoles à l'échelle de l'UE, cède le pas au pluralisme à la fin des années 1970. La position hégémonique des organisations professionnelles s'effrite sous la pression de plusieurs facteurs, notamment l'entrée dans l'ère de la globalisation et le tournant néolibéral européen (Jobert, 1994). Toutefois, l'évolution des modes de représentation des intérêts se fait avant tout dans la continuité pour les organisations de la filière sucre: les relations privilégiées construites pendant la période de cogestion se font plus confidentielles. Ainsi les lieux de discussion formels entre les professionnels et la Commission demeurent importants, mais le carnet d'adresses des professionnels du sucre semble l'être davantage.

\subsection{L'implantation ancienne des professionnels du sucre à Bruxelles comme atouts politiques}

Si les modes de représentation de la filière sucre n'ont pas été bouleversés par le système communautaire d'intermédiation des intérêts, néanmoins, à partir des années 
1980, la Commission élargit sa consultation et les organisations agricoles perdent l'hégémonie dont elles bénéficiaient à Bruxelles. Les problématiques agricoles ne sont plus seulement traitées par la DG Agri, d'autres directions comme la DG Santé des consommateurs, la DG Commerce, interviennent également dans les négociations. Alors que le CEFS et la CIBE avaient fait de la DG Agri leur interlocuteur privilégié, ils se voient contraints de nouer des liens avec certaines DG et certains groupes avec qui ils ne se trouvent pas en position de force. En outre, la saturation du marché communautaire et les politiques d'importation de sucre vis-à-vis des pays en développement viennent bouleverser la position privilégiée de la filière européenne du sucre. L'émergence de ladite mondialisation offre une place prépondérante à des interlocuteurs internationaux, comme l'Organisation mondiale du commerce (OMC), qui jouent un rôle essentiel dans les négociations agricoles. Enfin, le changement de discours sur la PAC introduit par la Commission Delors stigmatise la DG Agri, l'accusant d'être en partie responsable des dérives budgétaires agricoles. A travers elle, c'est également des groupes tels que la CIBE et le CEFS qui sont visés.

Malgré tout, ces transformations n'ont pas abouti à une remise en cause profonde des relations de proximité qui ont lié et lient encore les représentants de la filière sucre et les décideurs européens. Si l'ouverture de la Commission européenne à d'autres groupes est avérée, dans la mesure où les représentants des consommateurs, des associations environnementales ou des syndicats dits minoritaires sont invités et écoutés par les membres des instances décisionnelles européennes, la remise en cause de la position hégémonique de la CIBE et plus encore du CEFS n'est qu'apparente ${ }^{4}$ : les représentants de la filière vont continuer de privilégier les stratégies internes (Grossman et Saurugger, 2006) pour représenter et défendre leurs intérêts, et notamment les relations privilégiées qu'ils entretiennent historiquement avec les décideurs.

Certes, c'est bien à cette période que le CEFS et la CIBE commencent à recruter davantage de salariés afin qu'ils produisent des analyses économiques et qu'ils les diffusent largement auprès des instances européennes. Avant les années 1980, seules quelques grandes figures représentaient la filière. Ces nouveaux recrutements marquent l'adaptation de la filière au pluralisme et à la multiplication des lieux de discussions à l'échelle de l'UE. Les lobbyistes doivent occuper la scène politique et ne pas abandonner la place à leurs adversaires politiques. Mais si le nombre de lobbyistes a augmenté, il reste toutefois assez restreint puisqu'à peine une quinzaine de professionnels, toutes tâches confondues, se charge de représenter les intérêts du secteur. Agronomes spécialisés en économie, ou économistes, ces derniers témoignent en outre d'un certain attachement à la filière: le turn-over y est limité. Ces lobbyistes vont calquer leurs stratégies d'influence sur celles de leurs ainés, et la constitution du carnet d'adresses devient dès lors la stratégie d'influence par excellence.

C'est aussi à cette époque qu'on observe une multiplication des migrations du secteur public national et/ou européen vers les organisations du secteur sucre. Ce

\footnotetext{
${ }^{4}$ Les travaux de R. Balme et D. Chabanet (2001) sur le dialogue social européen supposent également la persistance de formes de néo-corporatisme au niveau européen.
} 
phénomène, qualifié aussi de pantouflage (Bauer et Bertin-Mourot, 1997), permet aux organisations professionnelles du sucre d'internaliser un carnet d'adresses et d'en faire profiter la filière. Ainsi, au début des années 2000, la migration du responsable sucre de la Direction des politiques économiques internationales (DPEI-Ministère de l'agriculture) en France vers le CEFS met en évidence les multiples réseaux d'influence dont bénéficient les fabricants de sucre. Ce fonctionnaire français était chargé à la DPEI de la politique du sucre. Il a quitté son poste pour une fonction de lobbyiste dans un groupe d'intérêt transnational. De même, le syndicat professionnel des fabricants de sucre (SNFS) est présidé, à partir de 2001, par Phillipe Soubestre. La trajectoire socioprofessionnelle de ce dernier met en évidence la puissance des fabricants de sucre français et l'influence dont ils bénéficient au niveau européen puisque P. Soubestre a commencé sa carrière à la Commission en 1963, et, après avoir bénéficié de plusieurs promotions, devient directeur général de la DG du service commun de gestion de l'aide communautaire aux pays tiers, pour occuper ensuite, à la Commission européenne, le poste de directeur général adjoint de la Direction générale du Développement. Il a occupé plusieurs postes dans le domaine du développement et a été membre du cabinet du Commissaire Jean-François Deniau et chef de cabinet du Commissaire Claude Cheysson (Dimier, 2003). Depuis 2001, il a quitté la fonction publique européenne et a présidé, entre autre, le SNFS et l'Association nationale de l'industrie agro-alimentaire France (ANIA). Les fonctions que le président de la SNFS a occupées au sein de la Commission mettent en évidence l'importance des enjeux traités au sein du SNFS, l'influence et le prestige de ce syndicat. Les compétences relationnelles, procédurales et institutionnelles acquises lors de sa carrière européenne sont réinvesties sur le plan national. Ces migrations d'agents de la haute fonction publique nationale ou européenne vers le secteur privé européen ou national suggèrent que ces derniers puissent profiter d'un accès privilégié aux instances de décision. Au-delà des proximités sociales et du carnet d'adresses dont disposaient les professionnels du sucre dans les années 1980 et 1990, ce sont aussi des visions du monde qui s'échangeaient.

\subsection{La circulation des idées et le partage de cadre d'interprétation du monde}

Au-delà des questions éthiques que posent ces migrations, notamment en termes de collusion d'intérêts, les travaux de Y. Dezalay et B. Garth (2002), qui mettent en évidence les interactions entre le «monde des exportateurs » nord-américains et les élites au pouvoir dans quatre pays d'Amérique latine, montrent que la circulation des élites favorise la circulation des idées et, plus précisément même, des cadres d'interprétation du monde au sein de structures différentes. La notion de réseau d'action publique (Le Galès et Thatcher, 1995) rend possible l'étude de la fabrication de sens de l'action publique (Hassenteufel et Smith, 2002) et de fait la circulation des idées. En s'intéressant aux discours des acteurs et à leurs positions politiques, on remarque que les cadres d'interprétation du problème portés par la CIBE et le CEFS et ceux des décideurs européens convergent. Tous, à travers les individus qui les représentent, affichent un cadre d'interprétation du monde identique, à savoir le référentiel du marché. Comme les fonctionnaires européens, les représentants de la filière ont analysé la situation en des termes économiques, comme l'illustrent les propos suivants : 
Entretien DG Agri, 2005: "Vues les contraintes du marché international, on ne peut pas faire autrement, ce seront forcément les plus compétitifs qui devront aller sur le marché mondial, les français, les allemands... Il faut arrêter de leur faire profiter de protections très très fortes comme ça a pu être le cas, il faut qu'ils aillent un peu se mesurer aux autres, sans distorsions incroyables, et ils ont en sont capables. En tout cas ceux qui en sont capables doivent aller sur le marché mondial, les autres..., les autres doivent se reconvertir... »

Les représentants de la filière ne remettent pas en cause les cadres d'interprétation proposés par les décideurs européens, ils partagent le même référentiel cognitif orienté vers la compétitivité et la libéralisation plus ou moins contrôlée des échanges mondiaux. De plus, le "pantouflage », l'ancienneté des relations entre les représentants de la filière et les décideurs européens, et le carnet d'adresses dont ils disposent, continuent de faciliter la représentation des intérêts de la filière sucre.

En effet, si les proximités sociales entre les décideurs européens et les professionnels du sucre ont permis à ces derniers de se construire un carnet d'adresses assez facilement jusqu'aux années 1980, ce processus a été rendu possible parce que l'univers professionnel du lobbying à cette période était moins compétitif et moins dense qu'aujourd'hui. Les professionnels les plus anciens ont commencé à construire leur réseau et à développer leur capital social dans les années 1970 : ils ont bénéficié de contacts "montants » peu à peu dans la Commission à mesure qu'ils avançaient dans leur carrière. Toutefois l'univers professionnel dans lequel arrivent les jeunes lobbyistes aujourd'hui est différent, puisque bien plus dense et concurrentiel. On identifie en effet aujourd'hui plus de $15000^{5}$ lobbyistes dans la capitale bruxelloise et le nombre d'organisations professionnelles, d'entreprises de consultance et de cabinet d'avocats approche les 3 000, ce qui tend à « anonymiser » davantage les nouveaux recrutés : leur carnet d'adresses parait plus complexe à construire. Epaulés par les plus anciens, ils tentent de se construire leur propre réseau de connaissances. Toutefois, à la différence des taux de turn-over important observés au sein du COPA (Hrabanski, 2006), le nombre restreint de professionnels du lobbyisme dans la filière sucre tend plutôt à fidéliser les jeunes recrues. Celles-ci ont tout intérêt à profiter du capital social de leurs aînés pour construire leur propre réseau professionnel, ce qui ne peut se faire que dans la durée.

Enfin, à partir des années 1990, le mode de représentation des intérêts prisé par la Commission semble accorder une place de plus en plus importante à l'expertise, offrant ainsi la possibilité aux professionnels du sucre d'intervenir en tant qu' « experts » dans le débat politique.

\footnotetext{
5 On ne connait pas le nombre exact de lobbyistes à Bruxelles, ce qui s'explique notamment par l'hétérogénéité de cette catégorie et aux modes de recensement. Les chiffres avancés viennent de l'article du journal Le Monde du 29/01/2006 «A Bruxelles, 15000 lobbyistes s'activent dans les coulisses des médias et de l'Union Européenne ", chiffres que confirme l'article plus récent de Adrien Sellez, dans eurosdwillage.eu, "Le temps de la transparence pour les lobbyistes bruxellois? ", bttp://www.eurosduvillage.eu/2009-annee-de-la-transparence-pour,2378. html
} 


\section{Les usages de l'expertise : entre lobbying et socialisation aux normes d'action publique}

Dans le secteur du sucre, les groupes consultatifs, en rassemblant des experts issus $\mathrm{du}$ monde professionnel, interviennent dans une phase cruciale du processus décisionnel : l'initiative et la formulation des problèmes publics (Robert, 2009). Pour participer à cette étape du processus décisionnel, les groupes d'intérêt se doivent, pour être entendus, de proposer des expertises et peuvent de cette façon représenter leurs intérêts. L'expertise apparait ici non seulement comme un mode de représentation des intérêts (Saurugger, 2002), mais également comme un registre rhétorique auquel doivent souscrire les groupes afin de s'imposer comme interlocuteur légitime (Robert, 2010). Les observations réalisées pendant la tenue des groupes consultatifs entre 2005 et 2006, à la DG Agri, et la réalisation de près de dix entretiens semi-directifs effectués auprès des professionnels du sucre et de plusieurs «eurofonctionnaires » a permis d'examiner comment les lobbyistes de la filière sucre participent, via l'expertise, au processus décisionnel européen. Leurs activités professionnelles s'adaptent aux demandes de la Commission et ils conforment leurs pratiques aux attentes institutionnelles.

\subsection{L'utilisation ambiguë de l'expertise à l'échelle de l'UE : déroulement et enjeux des groupes consultatifs « sucre»}

Le recours à l'expertise renvoie à des situations très hétérogènes. Les instances décisionnelles européennes semblent particulièrement enclines à employer ces notions. Le recours aux experts est extrêmement courant à l'échelle de l'UE, tant au moment de la consultation que de la prise de décision et de l'évaluation. Le nombre de sujets et de domaines politiques ne cesse de croître et les élites politiques justifient de plus en plus leurs décisions grâce au travail des experts. Plus encore, Cécile Robert (2001) a montré que les fonctionnaires européens et la Commission établissent une opposition entre ce qui est politique et ce qui est technique. Ces derniers assimilent ce qui est politique aux enjeux nationaux, ce qu'ils opposent à la dimension technique caractérisée par la neutralité, l'indépendance, la technicité et l'expertise... L'utilisation de l'expertise et de la légitimité des experts est, à ce titre, particulièrement ambiguë à l'échelle de l'Union européenne (Robert, 2010). On remarque un premier glissement du sens du mot « expertise » puisque, pour la Commission européenne, les groupes d'intérêt concernés par les orientations agricoles pourraient être à même de proposer une expertise. Les groupes seraient-ils capables de prendre un recul si important qu'il leur permettrait d'adopter un regard neutre sur les situations agricoles? L'observation des groupes consultatifs du sucre permet d'analyser en quoi consiste le rôle d'experts qui est assigné aux professionnels du sucre.

Près de vingt-huit « experts » issus de la production, huit issus de l'industrie de fabrication du sucre, huit autres issus du commerce, un représentant des salariés, et neuf membres de la Commission (DG Agri et Commerce) se retrouvent une fois par trimestre pour «expertiser» ensemble les orientations de la filière. L'importance numérique des «eurofonctionnaires » et la présence d'un chef d'unité soulignent l'attention que l'administration communautaire accorde au secteur du sucre, à la 
différence de l'intérêt porté à d'autres secteurs plus marginaux (Hrabanski, 2010). De même, l'assiduité de l'ensemble des «experts » atteste de l'intérêt que ces derniers portent aux groupes consultatifs. L'enquête par observation au sein des groupes montre, en outre, que ces groupes d' « experts », tout en étant policés, sont traversés par des rapports de force qui opposent les fonctionnaires à une partie des professionnels de la filière. Alors qu'un eurofonctionnaire s'exprimait pour obtenir l'avis de l'assemblée sur un point qu'il qualifiait de «technique », afin que soit décidé, à partir de cette information, le montant des aides, un des professionnels de la filière a refusé de lui fournir ce renseignement. Opposé aux modalités d'application de la réforme du sucre, alors en cours pendant l'observation (2006/2007), de par le préjudice qu'elle faisait peser sur certains producteurs, l'expert a alors expliqué au fonctionnaire qu'il ne jugeait pas opportun de lui transmettre cette information car la décision qui en découlerait, ne devait, selon lui, intervenir que bien plus tard bloquant, de cette façon, la prise de décision. Le fonctionnaire lui a alors rétorqué que la décision serait prise quand même et qu'il était donc dans leur intérêt de lui fournir l'information. Ce professionnel du lobbyisme, soutenu par le lobbyiste du CEFS, a refusé. Cet échange révèle ainsi les rapports de force qui existent entre les «experts » et les membres de la Commission, et aussi la forme des échanges entre les deux parties. Chacun est resté cordial et très calme, plongeant ainsi le débat dans le domaine du «technique», chacun des intervenants appuyant sa position sur une argumentation précise et structurée.

Ainsi, au-delà de l'expertise fournie par les différents experts, ces groupes permettent aux lobbyistes du sucre d'y représenter leurs intérêts. Toutefois, le ton courtois des intervenants met en évidence l'ambiance policée et feutrée de ce type de réunion (Abelès et Bellier, 1996). En observant les prises de parole des experts, on décèle en effet leurs savoir-faire et savoir-être, ces derniers étant capables de se conformer au type d'échanges valorisés par la Commission, allant même parfois jusqu'à recourir à une rhétorique de type scientifique pour argumenter leur position.

\subsection{La technicité comme registre discursif au sein du groupe consultatif et la socialisation aux normes et standards d'action publique de l'UE}

Si les experts issus des groupes d'intérêt questionnent largement la portée réelle des groupes consultatifs, dans la mesure où la majeure partie des orientations leur semble avoir été déjà décidée, les lobbyistes y interviennent et endossent le rôle d'expert qu'on leur assigne en adoptant des savoir-faire et savoir-dire spécifiques. Ils y rencontrent les différents fonctionnaires européens de la division sucre, entretiennent officiellement avec eux des liens et s'assurent que les orientations ne vont pas à l'encontre de leurs intérêts et des positions émises officieusement lors de contacts informels. Ils endossent ainsi le rôle qu'on leur assigne en commentant des chiffres, en proposant des analyses sur la conjoncture des différents marchés nationaux et mondiaux et en s'échangeant des informations sur l'éventuelle portée d'une nouvelle mesure. C'est à cette occasion qu'ils tentent, d'une part, d'influencer les orientations qu'ils ont encore la possibilité de modifier et, également, de légitimer leur participation au processus décisionnel en prouvant leur crédibilité et leur capacité à se conformer au rôle d'expert qu'on attend d'eux. Ainsi au-delà du fait d'être écouté et d'avoir de l'influence, il s'agit 
surtout de s'imposer comme un acteur régulier et crédible, l'objectif est alors d'institutionnaliser un dialogue officiel.

La production d'une littérature scientifique, produite et qualifiée comme telle par les groupes d'intérêt, s'inscrit également dans cette dynamique. Si la Commission dispose de sa propre expertise scientifique et fait le cas échéant appel à des cabinets spécialisés pour évaluer l'impact des mesures, les groupes d'intérêt ne se privent pas de lui soumettre des comptes rendus et analyses sur les thèmes qui leur semblent cruciaux. Les codes rhétoriques et l'attention portée à la neutralisation des positions doivent participer à faire reconnaitre leur groupe d'intérêt communautaire comme un acteur crédible. Le recours à l'économie dans ses utilisations les plus pointues ( diagrammes, tableaux d'analyse sectorielle, ...), l'organisation des livrets blancs et livrets verts selon des codes particuliers, propres au monde scientifique et universitaire, d'où est issue la majorité des fonctionnaires, permettent aux groupes d'intérêt d'assurer aux fonctionnaires qu'ils font bien partie du monde sacré, par opposition à ceux qui appartiendraient au monde profane et qui refuseraient de se prêter aux codes et rituels de l'administration. Les experts en retour démontrent ainsi leur légitimité cognitive (Camau et Massardier, 2009) à travers l'utilisation de la technicité et le recours aux terminologies propres aux sciences économiques. Le recours à la technique et à la science comme registre de justification vient ainsi confirmer le «faisceau des pratiques» (Hugues, 1958) du lobbying, questionnant le rôle de la science dans la professionnalisation de la vie politique.

Mais surtout en organisant la forme des échanges à travers le rôle d'expert qu'elle assigne aux participants, la Commission européenne développe ses capacités de production et de prescription de normes et de standards d'action publique. En adoptant la posture de l'expert, les représentants de la filière peuvent, d'une part, représenter leurs intérêts en soutenant des positions qui s'appuient sur des expertises «scientifiques » et, d'autre part, attestent qu'ils maitrisent bien les codes rhétoriques, autrement dit «les bonnes pratiques » promues par l'UE : l'adoption de ces codes rhétoriques renvoie à un phénomène plus large de socialisation à ce que doit être «la politique » à l'échelle de l'UE. Autrement dit, en organisant la forme des échanges à travers le rôle d'expert qu'elle assigne aux participants, la Commission européenne développe ses capacités de production et de prescription de normes et de standards d'action publique. Ainsi, loin d'une certaine forme de privatisation de l'action publique (Demortain, 2005), l'article a au contraire montré comment les instances de l'UE, et notamment la Commission, invitent indirectement les groupes d'intérêt européens à suivre "ces bonnes pratiques ", gouvernant ainsi à distance (Le Galès et Scott, 2008). De cette façon, la Commission agit en déterminant des standards et les paramètres de l'action publique, plutôt qu'en intervenant directement. Les groupes d'intérêt de la filière sucre, en quête de crédibilité, anticipent alors les attentes informelles de la Commission.

\section{Conclusion}

L'article analyse l'évolution des pratiques de lobbying auprès des instances européennes du début du $\mathrm{XX}^{\mathrm{e}}$ siècle jusqu'à aujourd'hui. L'étude des modes de représentation des intérêts des organisations professionnelles de la filière sucre dessine 
trois périodes distinctes. La première montre comment l'organisation internationale des betteraviers européens (CIBE) s'est structurée et a représenté les intérêts de la filière à l'échelle de l'Europe, auprès des instances décisionnelles internationales, notamment de la SDN, avant même la structuration politique de l'UE. L'analyse des archives souligne la proximité idéologique et sociale entre l'élite agricole et les décideurs politiques nationaux et internationaux de l'époque. Cette première période a jeté les bases de la période suivante, qui commence au moment de la création de la CEE et qui se caractérise par une cogestion forte entre les instances communautaires et les représentants de la filière. Ainsi du début du XXe siècle jusqu’à la fin des années 1970, la cogestion du secteur s'est faite en collaboration étroite avec les betteraviers et les fabricants de sucre. La légitimité historique, l'ancrage territorial, la proximité idéologique et les liens interpersonnels qui unissaient les décideurs européens et les représentants offraient à ces derniers un accès privilégié au processus décisionnel et leur permettaient de diffuser largement leur position.

La troisième période s'intéresse aux transformations du lobbying à partir des années 1980, au moment de l'entrée dans l'ère de la globalisation et du pluralisme. Malgré les évolutions du système d'intermédiation des intérêts, les représentants de la filière vont poursuivre leur stratégie d'influence, basée notamment sur les relations privilégiées qu'ils entretiennent avec des représentants de la Commission. Cette période souligne ainsi l'importance du carnet d'adresses dans les processus politiques, mais aussi les difficultés, pour les plus jeunes lobbyistes de se construire ce carnet d'adresses, dans un univers professionnel qui a vu ses effectifs exploser depuis les années 1960. Cette dernière période semble se caractériser également par la montée en puissance d'une certaine technicisation des débats à travers le recours à l'expertise par la Commission européenne. L'article a ainsi montré que l'expertise fait partie des différentes pratiques de lobbying, mais surtout souligne comment cette forme de lobbying est largement produite et encouragée par les instances de l'UE. Celles-ci, en produisant des normes et des standards d'action publique, orientent les pratiques de lobbying menées par les groupes. Ce résultat pousse à analyser le lobbying non seulement comme un faisceau de pratiques menées par des acteurs spécifiques dotés d'une culture organisationnelle et de ressources politiques propres, mais surtout ces pratiques doivent être analysées à la lumière de l'interaction entre ces groupes d'intérêt et les instances décisionnelles, en tenant compte de leur genèse et de leur institutionnalisation. Enfin, la perspective diachronique adoptée montre à quel point l'ancrage historique est déterminant pour les organisations mobilisées à Bruxelles, et pose d'autant plus la question de l'accès aux ressources des groupes d'intérêt à l'échelle de l'UE.

\section{Bibliographie}

Abelès M., Bellier I. (1996) La Commission européenne du compromis culturel à la culture politique du compromis, Revue français de science politique 46, 431-456.

Ayberk U., Schenker F.-P. (1998) Des lobbies européens entre pluralisme et clientélisme, Revue française de science politique 48 (6), 725-755. 
Balme R., Chabanet D. (2001) Dialogue social européen et transformation des négociations collectives, Politique européenne 4, 119-139.

Barjot D. (1994) Internationale Cartels Revisited-Vues nowvelles sur les cartels internationaux 1880-1980, Caen, Editions du lys, 383 p.

Barral P. (1968) Les agrariens français de Méline à Pisani, Paris, Armand Colin, 386 p.

Bauer M., Bertin Mourot B. (1997) La tyrannie du diplôme initial et la circulation des élites: la stabilité du modèle français, in: Le recrutement des élites en Europe, Suleiman E., Mendras H. (dir.), Paris, Editions La Découverte, 48-63.

Boursier A. (1983) L'bistoire de la betterave, Paris, Editions SEDA, 223 p.

Buissière E. (2006) Enjeux européens et lobbying, in: Industire et politique en Europe occidentale et aux Etats-Unis: XIX ${ }^{e}$ et $X X^{e}$ siècles, Paris, Presses Paris Sorbonne, 403-408.

Camau M., Massardier G. (2009) Introduction, in: Démocraties et autoritarismes. Fragmentation et bybridation des régimes, Camau M., Massardier G. (eds), Paris, Karthala, 7-43.

Cayre H. (1982) Annexes, in: Histoire de la CIBE, Paris, Editions SEDA, 46 p.

Commission européenne (2001) La gouvernance européenne : un livre blanc, 46 p.

Della Porta D., Tarrow S. (2005) Transnational Protest and Global Activism, Oxford, Rowman and Littlefield Publishers, 304 p.

Delorme H. (2002) Les agriculteurs et les institutions communautaires: du corporatisme agricole au lobbyisme agro-alimentaire, in: L'action collective en Europe, Balme R., Chabanet D. et Wright V. (eds), Paris, Presses de Sciences Po, 313-346.

Demortain D. (2005) Le lobbying à Bruxelles ou la politisation comme métier (observation), Terrains et travaux 1, 34-52.

Dezalay Y., Garth B. (2002) La mondialisation des guerres de palais, Paris, Seuil, 504 p.

Dimier V. (2003) Institutionnalisation et bureaucratisation de la Commission européenne: l'exemple de la Direction générale du Développement, Politique européenne 3, 99-121.

Fouilleux E. (2003) La politique agricole commune et ses réformes, Paris, l'Harmattan, 403 p.

Freidson E. (1998) Professionalism reborn. Theory, prophecy and policy, Chicago, University of Chicago Press, $248 \mathrm{p}$.

Grossman E., Saurugger S. (2006) Les groupes d'intérêt. Action collective et stratégies de représentation, Paris, Armand Colin, 251 p.

Hassenteufel P., Smith A. (2002) Essoufflement ou second souffle? L'analyse des politiques publiques «à la française ", Revue française de science politique 52-1, 53-73.

Hervieu B. (1996) Les agriculteurs, Paris, PUF, 127 p. 
Hervieu B., Lagrave R.-M. (1992) Les syndicats agricoles en Europe, Paris, l'Harmattan, $318 \mathrm{p}$.

Hrabanski M. (2010) Les groupes d'experts de la Direction générale de l'Agriculture: diversité des usages de l'expertise et socialisation aux normes d'action publique de l'Union européenne, Politique européenne 32, 99-123.

Hrabanski M. (2006) Lobbyiste agricole, lobbyiste en agriculture. Recrutement et carrières des représentants d'intérêts du COPA, in: Lobbying et lobbyistes en Europe, Michel H. (éd.), Strasbourg, Presses universitaires de Strasbourg, 47-68.

Hugues E. (1958) Men and their Work, Westport, Connecticut, Greenwood Press, 184 p.

Jobert B. (1994) Le tournant néo-libéral en Europe. Idées et recettes dans les pratiques gouvernementales, Paris, L'Harmattan, $328 \mathrm{p}$.

Le Galès P., Scott A.J. (2008) Une révolution bureaucratique britannique? Revue française de sociologie 49, 301-330.

Le Galès P., Thatcher M. (1995) Les réseaux de politique publique, Paris, L'Harmattan, 278 p.

Michel H. (2005) Un groupe en pratiques, les pratiques d'un groupe, in: Lobbyistes et lobbying de l'Union européenne, Strasbourg, Presses universitaires de Strasbourg, 9-21.

Michel H. (2003) Pour une sociologie des pratiques de défense : le recours au droit par les groupes d'intérêts, Sociétés contemporaines 52, 5-16.

Muller P. (2000) Analayse cognitive des politiques publiques: vers une sociologie politique de l'action publique, Revue français de science politique 50, 189-208.

Muller P. (1980) Le technocrate et le paysan, Paris, Editions ouvrières, 174 p.

Pesche D. (2000) Le syndicalisme agricole spécialisé en France, Paris, L'Harmattan, 368 p.

Pesche D., Hrabanski M. (2010) Défendre un produit. Entre logiques politiques nationales et enjeux globaux, in: Les mondes agricoles en politique, Hervieu B., Mayer N., Muller P., Purseigle F. et Rémy J. (éds), Paris, Presses de Sciences-Po, 273-291.

Pinçon M., Pinçon-Charlot M. (1997) Voyage en grande bourgeoisie, Paris, PUF, 192 p.

Richardson J.D. (1993) Pressure groups, Oxford, Oxford University Press, 288 p.

Robert C. (2010) Les groupes d'experts dans le gouvernement de l'Union européenne. Bilans et perspectives de recherche, Politique européenne 32, 7-38.

Robert C. (2009) Les eurofonctionnaires et leurs experts. Stratégies de recrutement et modalités d'encadrement des groupes d'experts européens, in: Démocraties et autoritarimes. Fragmentation et bybridation des régimes, Camau M., Massardier G. (éds), Paris, Karthala, 287-303.

Robert C. (2001) La Commission européenne dans son rapport au politique : pourquoi et comment faire de la politique sans en avoir l'air? Pôle Sud 15, 61-75. 
M. Hrabanski - Revue d'Etudes en Agriculture et Environnement, 92 (2), 143-160

Saurugger S. (2002) L'expertise: un mode de participation des groupes d'intérêt au processus décisionnel communautaire, Revue française de science politique 52, $375-401$.

Servolin C. (1989) L'agriculture moderne, Paris, Seuil, 318 p.

Wagner A.-C. (1998) Les nowvelles élites de la mondialisation, Paris, PUF, 236 p. 